\title{
Study of Various Winding Topologies for Self-Shielding Induction Cookers
}

\author{
Christophe Auvigne *, Florian Copt *, Christophe Winter *, and Yves Perriard *
}

\begin{abstract}
In this paper, the magnetic coupling between an induction cooker and a metallic pot is studied for various winding topologies. Effects of the winding orientation on the power transfer capability and on the emitted magnetic field under the cooker are discussed and compared. A novel topology inspired by permanent magnet Halbach array is proposed. This consists in coupling in both horizontal and vertical directions and to create an asymmetric field in order to reduce the emission under the cooker.
\end{abstract}

\section{Keywords: Contactless power transfer, Transformer, Winding topology, Eddy Currents, Induction heating}

\section{Introduction}

Induction cookers have become a standard in consumer market of home and professional kitchen. Magnetically induced currents heat up any conductive target placed in the radiated field. For cooking applications, the involved power is in the range of $1.5 \mathrm{~kW}$. To be able to transfer this range of power, dedicated ferromagnetic stoves and pans have been commercialized to ensure sufficient coupling with the cooker [1]. However, existing kitchen utensil, such as aluminium Italian coffee maker for example, cannot be used anymore as it in a refurbished kitchen.

This paper is focused on possible ways to increase the magnetic coupling between a metallic pot and a cooker whilst reducing the radiated emitted magnetic field around the cooker. Given this frame, five winding topologies presented in Fig. 1 are compared. The first topology (a) is a conventional winding surrounding a ferrite plate. This is the most used topology in the literature [2]-[3]. The next two topologies $(b-c)$ represent a winding around the ferrite plate leading to a magnetic field horizontally oriented in the ferrite plate. The last two topologies (d-e) are inspired on Halbach array which is a known arrangement of permanent magnets that modifies the distribution of the magnetic field [4]. The aim of such a topology is to create a nonsymmetric field that reduces the emitted field under the cooker in order to be compliant with the EMF exposure

* Institute of Microengineering (IMT), Integrated Actuators Laboratory (LAI), Ecole polytechnique fe'de'rale de Lausanne (EPFL), Switzerland. (christophe.auvigne@epfl.ch, christophe.winter@epfl. ch, yves.perrird@epfl.ch)

Received 15 August 2013; $\quad$ Accepted 10 October 2013 guidelines drawn by the ICNIRP [5]. Moreover, the upper radiated field has bothvertical and horizontal coupling components which increase the magnetic coupling between the transmitter and the pot.

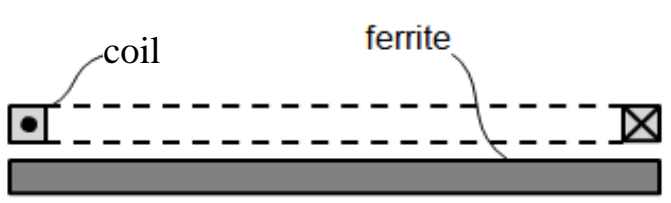

(a)

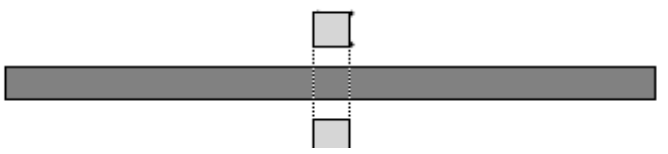

(b)

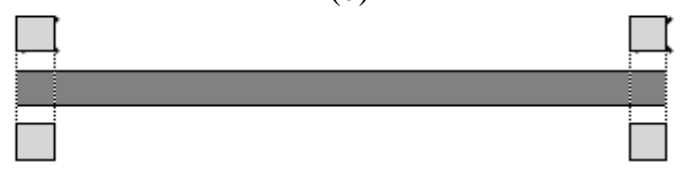

(c)

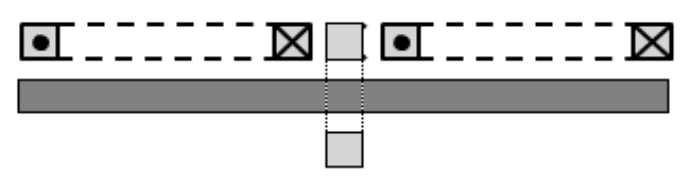

(d)

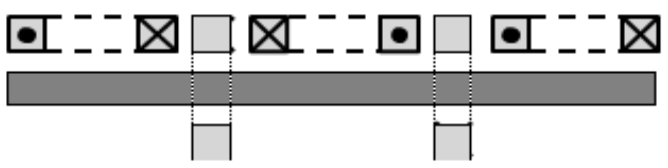

(e)

Fig. 1. Studied winding topologies (cross-section) 
As a first step, a simple electrical modeling of the transmitter and the pot is made. The equivalent impedances and their associated parameters are highlighted. Then, to obtain comparable results, the five topologies are designed to transfer $1 \mathrm{~kW}$ to the pot. The power transfer capability and the required copper volume are investigated for both iron pot and aluminum one. Then, the emission of the radiated magnetic field for different distances from the emitter is compared.

Fig. 2 presents the used Finite Element (FE) model (for the particular case of topology e) that is solved using the commercial software FLUX2D. As the heating Eddy currents are induced in the pot, a particular attention has been taken for the mesh of this element in order to take into account the skin effect and model accurately the current distribution inside the pot. The working frequency of the cooker has been set to $50 \mathrm{kHz}$.

Based on FE simulations, the equivalent electrical impedance brought back to the primary side of the cooker is evaluated. Since no other losses than Eddy current Joule losses are evaluated, the needed current to obtain a power of $1 \mathrm{~kW}$ in the pot can be evaluated by identification of the equivalent electrical resistance of the pot at the primary side and the simulation run again to evaluate the radiated magnetic field along the measurement line.

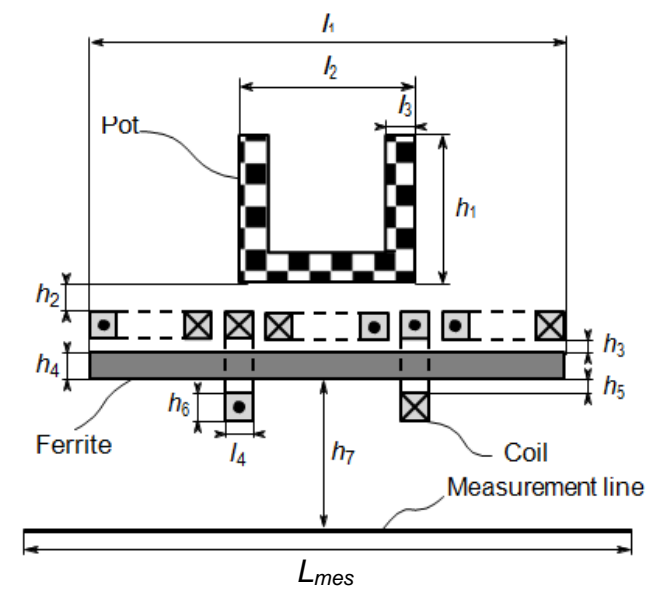

Fig. 2. Scheme of the FE model for the particular case of topology (e)

\section{Electrical Modeling}

An alternative magnetic field is generated with the coil(s). Eddy currents are generated in the metallic parts. The first Maxwell equation gives [6]:

$$
\nabla \times \vec{H}=\vec{J}+\frac{\partial \vec{D}}{\partial t}
$$

Table 1. Geometrical dimensions of the FE model for the initial topologies

\begin{tabular}{|c|c|}
\hline Dimension & Value $[\mathrm{mm}]$ \\
\hline$l_{1}$ & 150 \\
\hline$l_{2}$ & 100 \\
\hline$l_{3}$ & 2 \\
\hline$l_{4}$ & 7 \\
\hline$h_{1}$ & 100 \\
\hline$h_{2}$ & 5 \\
\hline$h_{3}$ & 2 \\
\hline$h_{4}$ & 5 \\
\hline$h_{5}$ & 2 \\
\hline$h_{6}$ & 3.6 \\
\hline$h_{7}$ & $50,100,150$ \\
\hline
\end{tabular}

The relation between the magnetic field and the induced current density for quasi static mode becomes

$$
\nabla \mathrm{x} \vec{H}=\vec{J}
$$

Due to skin effect, the current is concentrated to the surfaceof the plate. The skin layer thickness $\delta$, given in (3) is function of the magnetic permeability $\mu$, the electrical resistivity $\rho$ and the pulsation $\omega$ [7].

$$
\delta=\sqrt{\frac{2}{\mu \rho \omega}}
$$

This parameter is reported for several materials of interest and a frequency $\mathrm{f}=50 \mathrm{kHz}$ in Table 2 . One can see that the skin depth $\delta$ is quite the same for aluminium and copper while it is about six times higher for a typical iron material.

Table 2. Skin depth for several material $(\mathrm{f}=50 \mathrm{kHz})$

\begin{tabular}{|c|c|}
\hline Material & Skin layer $\delta[\mu \mathrm{m}]$ \\
\hline Copper & 292 \\
\hline Aluminum & 367 \\
\hline Iron & 58 \\
\hline
\end{tabular}

The electric equivalent model of the system is shown in Fig. 3. It is composed of an equivalent resistance representing the intrinsic resistance of the coil $\mathrm{R}$ and the resistance of the pot seen by the coil $R_{\text {pot }}$ ). The Joule losses in the pot are determined in (4) where $\rho$ is the material electrical resistivity and $\mathrm{J}$ the current density determined by (2). The equivalent resistance of the pot can be directly deduced from (4), as done in (5). Finally, the equivalent inductance Leq is defined in (6). An analytical determination of this resistance for a simple geometry is 
proposed in [8]. However, this is identified using FE method in this paper.

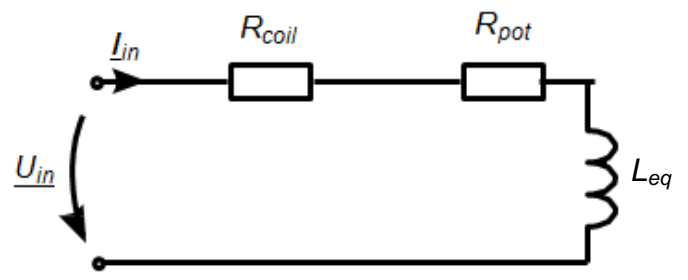

Fig. 3. Electric equivalent circuit

$$
\begin{gathered}
P=\int_{V} \rho J^{2} d V=R_{p o t} I_{\text {in }}{ }^{2} \\
R_{\text {pot }}=\frac{P}{I_{\text {in }}{ }^{2}} \\
L_{\text {eq }}=\operatorname{Im}\left\{\frac{U_{\text {in }}}{I_{\text {in } \omega}}\right\}
\end{gathered}
$$

\section{Description of the Winding Geometries Studied}

The five scenarios studied are illustrated in Fig. 1. As a first step, each coil is composed of one turn. The resistance Rpot is computed for the five scenarios in this configuration. The needed current in Amper - Turns to transfer $1 \mathrm{~kW}$ in the pot, can easily be deduced from the resistance Rpot. Based on the computed currents, the surface of the conductors needed to ensure a current density lower or equal than $3 \mathrm{~A} \cdot \mathrm{mm}-2$ can be evaluated. The maximal current density value has been chosen to avoid overheating keeping in mind that the fill factor of the actual winding can only be in the range of $50 \%$, according to [9]. These values are listed in Table 3. The windings are then redesigned in order to match the section previously defined. The adapted winding geometries for the five scenarios are shown at scale in Fig. 4. The resistances $R_{\text {pot }}$ and the needed currents are computed again $\left(R_{\text {pot }}\right)$ for the five scenarios with the new coils geometry. The total copper volumes $V_{C U}$ are also computed for the five scenarios (Table 4).

Table 3. Parameters evaluation with one-turn coils (iron pot)

\begin{tabular}{|c|c|c|c|}
\hline Scenario & $R_{\text {pot }}[\mathrm{m} \Omega]$ & $I_{\text {in }}[$ A.Turn] & section $\left[\mathrm{mm}^{2}\right]$ \\
\hline (a) & 4.4 & 478 & 159 \\
\hline (b) & 9.2 & 331 & 110 \\
\hline (c) & 0.7 & 1174 & 391 \\
\hline (d) & 77 & 114 & 38 \\
\hline (e) & 114 & 94 & 31 \\
\hline
\end{tabular}

The equivalent resistance $R_{\text {pot }}$ for one turn is maximum for the scenario (e). This result is intuitive since this topology has the biggest number of coils and the magnetic coupling between the windings and the pot is ensured with both tangential and normal component. Since the resistance of scenario (b) is bigger than scenario (a), the coupling path is better in scenario (b). This result has already been demonstrated for contactless power transfer purpose in [10] Even if the number of coils used in scenario (e) is bigger than the other ones, the total necessary volume of copper is smaller than for scenarios (a) and (c) and about the same for the other scenarios. The biggest equivalent resistance with the modified geometry is still obtained with scenario (e), which means that this topology offers the best magnetic coupling with the pot.

Table 4. Parameters evaluation with the adapted geometry (iron pot)

\begin{tabular}{|c|c|c|c|}
\hline Scenario & $R_{\text {pot }}[\mathrm{m} \Omega]$ & $I_{\text {in }}$ [A.Turn] & $\begin{array}{c}\text { Volume } V_{C U} \\
{\left[\mathrm{~cm}^{3}\right]}\end{array}$ \\
\hline (a) & 9 & 334 & 51 \\
\hline (b) & 7.7 & 361 & 24 \\
\hline (c) & 7 & 378 & 171 \\
\hline (d) & 70 & 120 & 30 \\
\hline (e) & 104 & 98 & 36 \\
\hline
\end{tabular}

Table 5. Parameters evaluation with the adapted geometry

(Al pot)
\begin{tabular}{|c|c|c|c|}
\hline Scenario & $R_{\text {pot }}[\mathrm{m} \Omega]$ & $I_{\text {in }}[$ A.Turn] & $P_{A L}[\mathrm{~W}]$ \\
\hline (a) & 0.29 & 334 & 32 \\
\hline (b) & 0.18 & 361 & 23 \\
\hline (c) & 0.15 & 378 & 22 \\
\hline (d) & 1.7 & 120 & 24 \\
\hline (e) & 2.7 & 98 & 26 \\
\hline
\end{tabular}

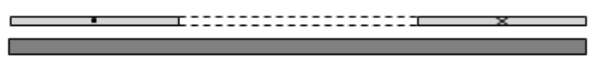

(a)

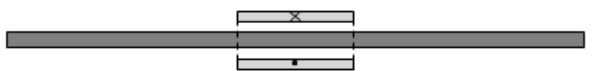

(b)

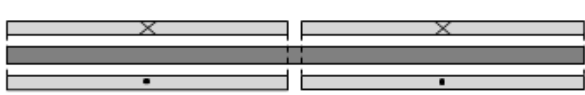

(c)

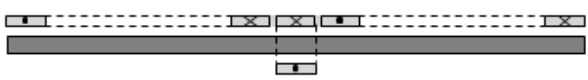

(d)

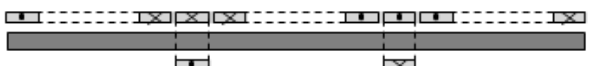

(e)

Fig. 4. Adapted topologies (cross-section)

The iron pot is replaced by an aluminium one and the 
simulations are computed again within the same conditions (current $I_{\text {in }}$ and geometries in Fig. 4). The equivalent resistance $R_{\text {pot }}$ and the transmitted power $P_{A L}$ are computed. The transmitted power using an aluminium pot is lower than the one using an iron one, which is consistent with (3). Although the transmitted power in this case is much lower, $R_{\text {pot }}$ is still higher for scenario (e) which confirms a better coupling for this topology.

\section{Study of the Radiated Magnetic Field}

For each scenario, the radiated magnetic field under the windings (on line Lmes in Fig. 2) is computed. The magnetic field modulus in function of the position on the line Lmes for a transmitted power of $1 \mathrm{~kW}$ are plotted for the five scenarios and at three different distances $h 7$ in Fig. 5, Fig. 6 and Fig. 7.

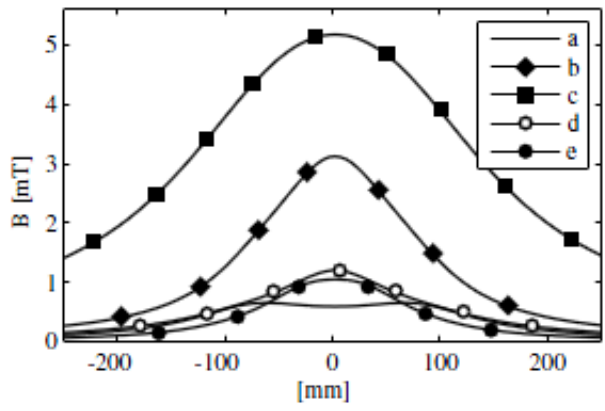

Fig. 5. $h_{7}=50[\mathrm{~mm}]$

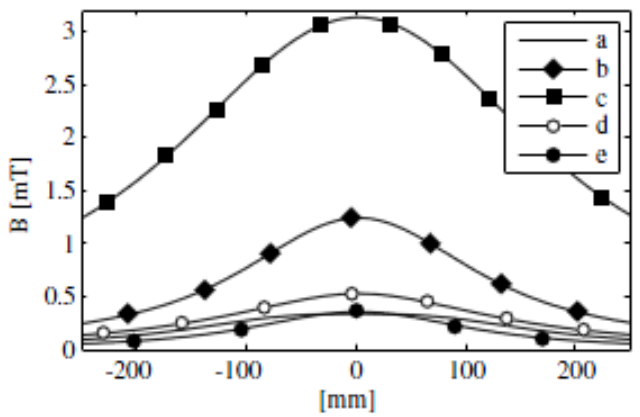

Fig. 6. $h_{7}=100[\mathrm{~mm}]$

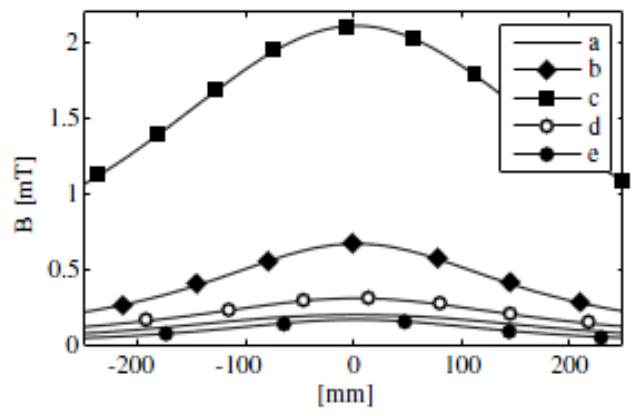

Fig. 7. $h_{7}=150[\mathrm{~mm}]$
One can see that whatever the measurement distances is, the scenarios (c) and (e) leads to the smallest radiated field while the scenario (c) leads to the largest one.

\section{Conclusion}

This paper focuses on magnetic coupling and power transfer capability for induction cookers purpose. In this way, five topologies of interest are studied and compared. The first one (scenario (a)) is the classical topology for contactless heating applications. The magnetic coupling is mostly ensured with the normal component of the magnetic field. The scenarios (b) and (c) couple the pot with the tangential component. The scenarios (d) and (c) are inspired from Halbach arrangement of magnets. This concept is applied with coils. An electrical modelling is provided in order to define the main parameters used for this study. For each scenario, the windings are computed to transfer a power of $1 \mathrm{~kW}$ to the pot with a current density of $3 \mathrm{~A}$ $\mathrm{mm}-2$ in order to avoid overheating of the coils.

The FE based simulations are used to compare the studied topologies regarding three factors: the power transfer capability (measured through Rpot), the radiated magnetic field and the required copper volume. Scenarios (d) and (e) lead to a higher equivalent resistance while scenarios (a) and (e) offer a smaller radiated field. The required copper volume for topology (e) is slightly smaller than topology (a).

As a result, the presented novel topology (scenario (e)) Increases the magnetic coupling between the transmitter and the pot. The equivalent resistance seen by the cooker is indeed increased. Moreover, the radiated magnetic field under the transmitter is decreased and the required copper volume is kept low. However, the main drawback is that the winding process complexity is increased compared to classical windings.

\section{References}

[1] M. E. Tulu and D. Yildirim, "Induction cooker design with quasi resonant topology using jitter drive method," in Environment and Electrical Engineering (EEEIC), 2013 12th International Conference on, pp. 1-6, 2013.

[2] L. Meng, K. W. E. Cheng, and K. Chan, "Heating performance improvement and field study of the induction cooker," in Power Electronics Systems and Applications, 2009. PESA 2009. 3rd Inter- national Conference on, pp. 1-5, 2009.

[3] J. Acero, O. Lucia, I. Millan, L. Barragan, J.-M. Burdio, and R. Alonso, "Identification of the material properties used in domestic induction heating appliances for system-level simulation and design purposes," in Applied Power Electronics Conference and Exposition (APEC), 2010 Twenty-Fifth Annual IEEE, pp. 439-443, 2010. 
[4] H. Li and C. Xia, "Halbach array magnet and its application to pm spherical motor," in Electrical Machines and Systems, ICEMS 2008. International Conference on, pp. 3064-3069, 2008.

[5] ICNRP, "Icnirp guidelines for limiting exposure to timevarying electric, magnetic and electromagnetic fields (up to 300 ghz), $" 1998$.

[6] M. Jufer, Traite' d'e'lectricite': e'lectrome'canique. Traite d'e'lectricite', Presses Polytechniques Romandes, 1995.

[7] R. Stoll, The analysis of eddy currents. Monographs in electrical and electronic engineering, Clarendon Press, 1974.

[8] M. Markovic and Y. Perriard, "Eddy current power losses in a toroidal laminated core with rectangular cross section," in Electrical Machines and Systems, 2009. ICEMS 2009. International Conference on, pp. 1-

[9] M. Budhia, J. Boys, G. Covic, and C.-Y. Huang, "Development of a single-sided flux magnetic coupler for electric vehicle ipt charging systems," Industrial Electronics, IEEE Transactions on, vol. 60, no. 1, pp. 318-328, 2013."

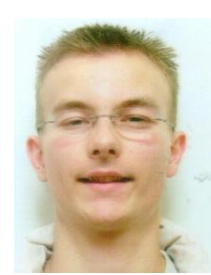

Christophe Auvigne was born in Ambilly, France, in 1987. He received the M. Sc. in Microengineering from the Swiss Federal Institute of Technology - Lausanne (EPFL) in 2011. He is currently a PhD student in the Integrated Actuator Laboratory at EPFL. His research interests are in the field of contactless power transfer and its associated power electronics

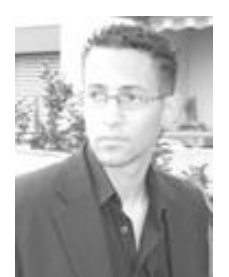

Florian Copt was born in Fribourg, Switzerland, in 1986. He received the M. Sc. in Microengineering from the Swiss Federal Institute of Technology - Lausanne (EPFL) in 2013. He is currently working at the Integrated Actuator Laboratory at EPFL. His research interests are in the field of contactless power transfer and its associated power electronics.

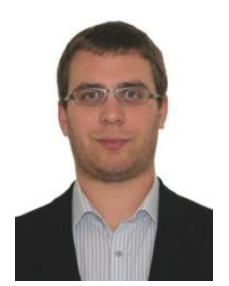

Christophe Winter was born in Bern, Switzerland, in 1986. He received the M. Sc. in Microengineering from the Swiss Federal Institute of Technology - Lausanne (EPFL) in 2009. He is currently a $\mathrm{PhD}$ student in the Integrated Actuator Laboratory at EPFL. His research interests are in the field of piezoelectric haptic actuators (mechanical and electronic drives design) and squeeze film modeling.

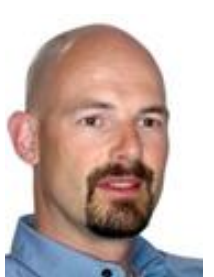

Yves Perriard was born in Lausanne in 1965. He received the $M$. Sc. in Microengineering from the Swiss Federal Institute of Technology - Lausanne (EPFL) in 1989 and the PhD. degree in 1992. Senior lecturer from 1998 and professor since 2003, he is currently director of the Integrated Actuator Laboratory at EPFL. His research interests are in the field of new actuator design and associated electronic devices. 